\title{
Testing of bus bar contact under non stationary current impacts
}

\author{
Amankeldy Bekbaev ${ }^{1, *}$, Erlan Sarsenbayev ${ }^{1}$, Vasiliy Titkov $^{2}$ \\ ${ }^{1}$ Satpaev Kazakh National Research Technical University, Almaty, Kazakhstan \\ ${ }^{2}$ Saint Petersburg State Polytechnic University,Saint-Petersburg, Russia
}

\begin{abstract}
This research paper is devoted to the analysis of non-stationary temperature field near plane bus bar contact. The possibilities of temperature and contact quality estimation by dynamic sensor on the accessible bus bar surface are showed. There is discussed the algorithm of temperature recognising directly on the contact surface. Numerical methods and analytical approaches are used.
\end{abstract}

\section{Introduction}

The operating mode of electrical installation determines thermal effect on current-carrying elements [1], and in particular on terminal connections, quality reduce of which can lead to serious consequences (welding, thermal breakdown). Short current pulses that occur in the busbarof power supply equipment as a result of, for example, motor start [2], disconnect of short circuits $[3,4]$, etc. can lead to a dangerous temperature rise in the contact surfaces of busbar.The available experimental findings on plane busbar heating by heavy short-time current describe the average busbar temperature, depending on its cross-section and configuration of the bolt holes [1]. Whereby, instant temperature value of direct-to-contact surface can not be fixed by any means of measurements due to its inaccessibility. At the same time instant temperature values in the area of local increase of the busbar resistivity (contact, holes) can be significantly higher than the average values. This fact is very important for the plane contact connections, changing its state under mechanical, environmental and long-term thermal factors leading to an increase oftransition resistance of the contact. Since the thickness of conduct busbar can be about dozen of millimeters, and the layer thickness with a high level of heat near the contact surface represents a very small fraction of a millimeter, then direct measurements on the busbar surface do not allow fixing directly the true value of the temperature in the contact zone.

However, analysis of the temperature field in the plane contact zone makes possible to establish functional temperature relationship in the contact zone and on the busbar surface, and also the relationship between fixing temperature on the busbar and physical characteristics of contact quality (frequency by transition resistance). Temperature determination of inaccessible surface (heat source plane) is reviewed in this paper. In case

* Corresponding author: bekbaev_a@mail.ru 
of stable algorithm for solving of the mentioned inverse problem under conditions of presence of interference and noise, programming of data communications in the controller that receives a signal in the form of timed sequence of temperature values on the busbar surface during the flow of current from the physical sensor, it allows to estimate the temperature and quality of contact in dynamics. It should be noted that using of dynamic temperature evaluation during impulse action as opposed to methods based on the analysis of stationary thermal field [5.6], makes it possible to observe the existence of defects under conditions of a more contrast temperature distribution for a certain length of time [7].

\section{Method}

As it is well known [8], the temperature field in a solid with heating source is described by the heat equation with respect to the temperature $T$

$$
\rho c \frac{\partial T}{\partial t}=\nabla \cdot(\lambda \cdot \nabla T)+\dot{q}
$$

Where $\rho, \mathrm{c}, \lambda$ are the density, specific heat and thermal conductivity, $t$ is time, volume power density of the distributed heat source. In case of heat analysis in the currentcarrying elements of installations the equation for volumetric source is:

$$
\dot{q}=\frac{|\bar{j}|^{2}}{\sigma}
$$

Where $\bar{j}$ is the vector of current density, $\sigma$ is conductivity. The characteristic time for distribution of the thermal field to a depth of $l$ is measured by parameter

$$
t_{D}=\frac{l^{2}}{D}
$$

Where $=\frac{\lambda}{\rho c}$. In particular, if characteristic dimensions in the plane of contact connections comprise several centimeters, then in case of copper busbar $(\lambda=400 \mathrm{~W} / \mathrm{m} \mathrm{K}, \rho$ $\left.=8960 \mathrm{~kg} / \mathrm{m}^{3}, \mathrm{c}=380 \mathrm{~J} / \mathrm{kg} \mathrm{K}\right) t_{D}$ is equal to few seconds. Therefore, during dynamic temperature assessment with the effects of current pulses with the time interval of $1000 \mathrm{~ms}$ the temperature field distribution from the contact zone along the busbar can be neglected.

Thus, the temperature field analysis in the direction perpendicular to the busbar plane at a specified limited time interval is to be performed with the use of one-dimensional heat equation, which is at constant thermal and physical characteristics of busbar material, has the form

$$
\frac{\partial T}{\partial t}=D \frac{\partial^{2} T}{\partial x^{2}}+\dot{q}
$$

Solution of this equation allows you to set up temperature relation in the contact plane $(x=0)$ and in the temperature measurement point $(x=l)$. Let us consider the distribution of heat release source. In the main cross- section of contacting busbars is taken a uniform distribution of current density $j$ and volumetric heat capacity (2).Despite the diversity of physical and mechanical processes occurring on the contact surface $[9,10]$ increased heat release occurs in the thin transition layer of the contact with the thickness $\Delta, \Delta<<l$, which has conductivity $\sigma_{1}$, lesser than the busbar material has $\sigma_{1}<\sigma$. Volume density of the heat capacity in the transition layer is also determined by the formula (2). However, since the 
thickness of the layer is smaller than the busbar thickness $l$, it is more convenient to consider heat generation in the layer in the form of heat flow at the contact boundary $(x=0)$

$$
F=-\left.\lambda \frac{\partial T}{\partial x}\right|_{x=0}=\frac{1}{2} j^{2}\left(\frac{1}{\sigma_{1}}-\frac{1}{\sigma}\right) \Delta
$$

The multiplier $1 / 2$ in the last formula takes into account that the heat in the transition layer is distributed by equal parts between the contact busbars.

For further analysis, it is advisable to write equations and ratios (1)-(3) in dimensionless variables: $\theta$ is a temperature, $\xi$ is a coordinate, $\tau$ is a time.

$$
\frac{\partial \theta}{\partial \tau}=\frac{\partial^{2} \theta}{\partial \xi^{2}}
$$

Despite the fact that actual forms of current pulses often have a oscillations form $[2,3,4]$ with its relatively short duration (from microseconds during short circuits up to units of seconds during starting currents) heat release can be represented by energy equivalent rectangular pulse of a similar duration.

In case of relatively long current pulses is $t_{p} \geq t_{D}$ indication of the active phase termination for the run of dependency $d T / d t$ is easily accomplished by the starting time of this dependence. For the described mode of problem solving for determining of the temperature of inaccessible surface can be made on the basis of analytical solutions for non-stationary thermal field in the plate created by a flat-source on one of its side, that is permissible with substantial degradation in contact quality when volumetric heat can be neglected in comparison with the heat flow arising in the transition layer. In this case, it is advisable to use the dimensionless form of equation of heat transfer that is proposed in [11].

According to [11] the following basic values can be selected: temperature $T_{b}=F_{0} l / \lambda$, where $F_{0}$ is constant flow on the surface $x=0$; time $t_{b}=l^{2} / D$. Then dimensionless temperature rise on the surface of the plate over the initial value $T_{0}: \theta=\left(T-T_{0}\right) / T_{b}$ are expressed by dependences [11]

$$
\begin{gathered}
\theta(0)=\tau+\frac{1}{3}-\frac{2}{\pi^{2}} \sum_{n=1}^{\infty} \frac{e^{-\pi^{2} 2 n^{2} \tau}}{n^{2}} \\
\theta(l)=\tau-\frac{1}{6}-\frac{2}{\pi^{2}} \sum_{n=1}^{\infty} \frac{e^{-\pi^{2} n^{2} \tau}}{n^{2}}(-1)^{n}
\end{gathered}
$$

Figure 1 shows the dependences calculated on the basis of formulas (7) depending on the dimensionless temperature at the contact surface $\theta(0, \tau)$ and the busbar surface $\theta(1, \tau)$, and the dependence

$$
f_{\theta}(\tau)=\frac{\theta(1, \tau)}{\theta(0, \tau)}=\frac{T_{1}\left(t / t_{b}\right)}{T_{0}\left(t / t_{b}\right)},
$$

Which allows us to express the contact surface temperature $T_{0}(t)$ by the measured temperature on the outer busbarsurface $T_{l}(t)$

$$
T_{0}\left(t / t_{b}\right)-T_{0}=\left(T_{1}\left(t / t_{b}\right)-T_{0}\right) f_{\theta}^{-1}\left(t / t_{b}\right)
$$




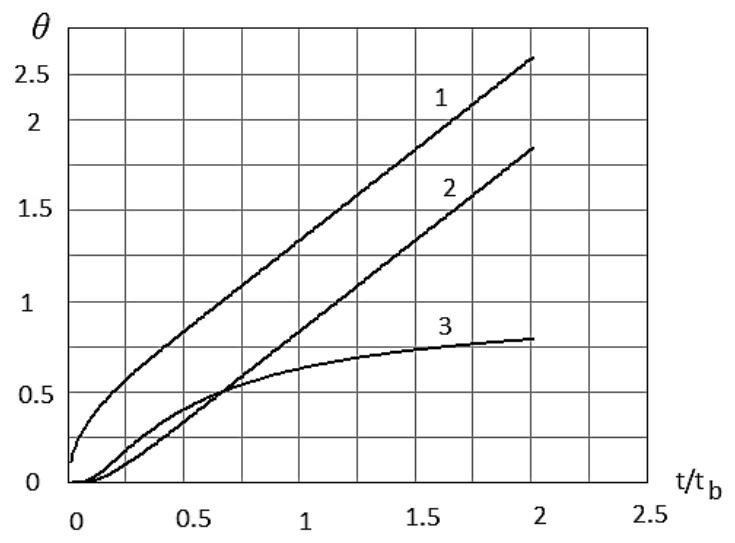

Fig. 1. Dimensionless time dependences of the contact temperature- 1 and busbar surface -2 , and the calibration curve $f_{\theta}(\tau)-3$.

Universal calibration function $f_{\theta}(\tau)$ can be stored in the memory of sensor controller as a sequence or analytic approximation. For example, in the area $2>\tau>0.1$ you can use the dependence in the form

$$
f_{\theta}(\tau) \cong 0.1082 \tau^{3}-0.5858 \tau^{2}+1.159 \tau-0.05514
$$

Thus, the contact surface temperature can be calculated from the temperature of busbar surface $T_{l}(t)$ by the expression

$$
T_{0}\left(t / t_{b}\right)=T_{0}(0)+\left(T_{1}\left(t / t_{b}\right)-T_{0}(0)\right) \cdot\left[0.1082\left(\frac{t}{t_{b}}\right)^{3}-0.5858\left(\frac{t}{t_{b}}\right)^{2}+1.159\left(\frac{t}{t_{b}}\right)-0.05514\right]^{-1}(10)
$$

The value of base time $t_{b}=l^{2} / D$, defined by the busbar thickness $l$, thermal conductivity of its material - D, can be included into the microcontroller program which carries out temperature calculation by the last expression. This circumstance determines the individual adjustment of the measuring device for each controlled contact. However, thicknesses and materials of busbars in power equipment do not vary significantly. In particular, materials are typically copper or aluminum, and the busbar thickness is in the range of 5-20 mm.

Therefore individual adjustment of sensor controller is not an intrinsic problem. The formula (10) used in the algorithm for temperature measure on busbar surface, can be applied only at the stage of temperature growth on contact surface. Therefore, obtained by means of (10) are important up to the biggest values of calculated sequence. Further behavior of analyzed dependencies can not be described by the Beck's formulas (7).Thus, the presented algorithm can estimate the maximum temperature reached by the contact surface.

Figure 3 and 4 show an example of the above described algorithm based on Beck's formulas (7) for the flat contact busbars of $5 \mathrm{~mm}$ thick during the break of short-circuit current at conduction of the transition layer contact equal to $0.1 \%$ from the conductivity of the base material. (Figure 3, 4). 


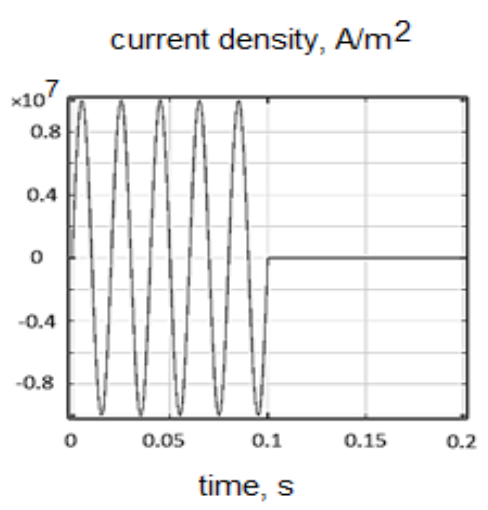

Fig. 3. Dependence of the current density at the contact area during the break of short circuit current.

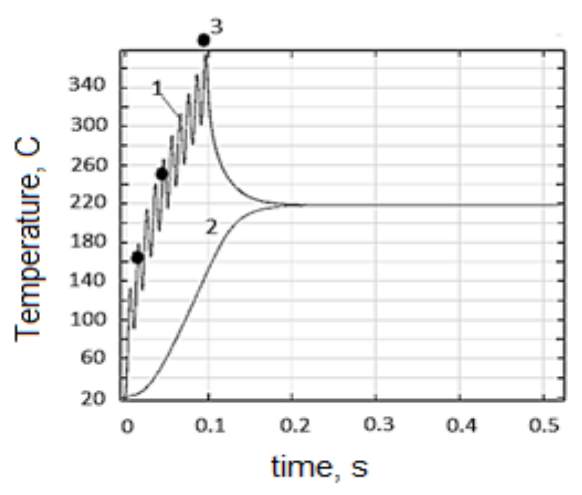

Fig. 4. The calculated temperature dependences from time in the contact surface -1 , on the busbar surface $-2,3$ - the contact temperature values, recovered by the formula (7).

It should be noted that, despite the differences of real conditions, characterized by pulsating with doubled industrial frequency heat nature of heat release, from the Beck's model, where it is assumed time - constant heat, the recovered temperature value of the contact surface are completely agreed with the calculated values.

\section{Conclusion}

The use of temporary temperature dependences, measured on the busbar surface for a short period of time, in order to neglect the influence of heat distribution along the busbar, make it possible to eliminate the need of consideration of external conditions for contact diagnosis. In case of quasi rectangular heat pulse in the contact the use of the same sensor controller, implementing the proposed algorithm, makes it possible to evaluate the value the maximum temperature direct on the contact surface. It must be noted that in this case there is no need to insert into the data processing algorithm of the temperature curve values on environmental parameters and structural features of the device. It is enough to insert data on the thickness and material of the busbar.

\section{References}

1. Y. Vishnevsky, Energoatomisdat, 728 (2002)

2. A. Ivanov, Publishing House 2, 532 (2006)

3. GOST R 52565-2006, Standartinform, 87 (2007)

4. GOST R 52736-2007, Standartinform, 40 (2007)

5. A. Nazarychev, F. Tadzhibaev, M. Sukhichev, V. Titkov, Energoexspert 2 (37), 12 (2013)

6. M. Sukhichev, V. Titkov, Electro 3, 17 (2010)

7. S.I. Krivosheev, A.I. Tajibav, V.V. Titkov, Scientific proceeddings XXIII 2, 165 (2015)

8. G.N. Aleksandrov, V.V. Borisov, V.L. Ivanov, Higher School, 312 (1985)

9. E. Kim, V. Omelchenko, S. Kharin, Bibliogr. 235, 230-232 (1977)

10. N. Myshkin, V. Konchits, M. Braunovich, Intellect, 558 (2008)

11. J. Beck, B. Blekuell, C. St. Clair, Mir, 313 (1989) 